\title{
Superconducting dipole magnet for the CBM experiment at FAIR
}

\author{
P. Kurilkin ${ }^{1, \star}$, P. Akishin ${ }^{1}$, A. Bychkov ${ }^{1}$, E. Floch ${ }^{1}$, Yu. Gusakov ${ }^{1}$, V. Ladygin ${ }^{1}$, A. Malakhov ${ }^{1}$, \\ G. Moritz ${ }^{2}, H$. Ramakers ${ }^{2}, P$. Senger ${ }^{2}, A$. Shabunov $^{1}, P$. Szwangruber $^{2}$, and $F$. Toral $^{3}$ \\ 1 JINR, Joliot-Curie 6, 141980, Dubna, Russia \\ ${ }^{2}$ GSI, Planckstrae 1, 64291 Darmstadt, Germany \\ ${ }^{3}$ CIEMAT, 28040, Madrid, Spain
}

\begin{abstract}
The scientific goal of the CBM (Compressed Baryonic Matter) experiment at FAIR (Darmstadt) is to explore the phase diagram of strongly interacting matter at highest baryon densities. The physics program of the CBM experiment is complimentary to the programs to be realized at MPD and BMN facilities at NICA and will start with beam derived by the SIS100 synchrotron. The 5.15 MJ superconducting dipole magnet will be used in the silicon tracking system of the CBM detector. The magnet will provide a magnetic field integral of $1 \mathrm{Tm}$ which is required to obtain a momentum resolution of $1 \%$ for the track reconstruction. The results of the development of dipole magnet of the CBM experiment are presented.
\end{abstract}

\section{Introduction}

The Compressed Baryonic Matter (CBM) will be one of the four major scientific experiments that are planned to be performed at the Facility for Anti-proton and Ion Research (FAIR) at Darmstadt, Germany [1]. The goal of the CBM research program is to perform the systematic investigation of the phase diagram of nuclear matter in the region of high baryon densities [2] and moderate temperature. These conditions will be created in the heavy-ion beam collisions with nuclear targets in the beam energy range from $2 \mathrm{AGeV}$ to $45 \mathrm{AGeV}$. The proton-nucleus interaction will be also studied. The start of CBM data taking is expected for 2021.

Earlier, the study of the QCD phase diagram at large baryon-chemical potentials have been curried out at AGS in Brookhaven [3] and at low CERN-SPS beam energies [4]. The STAR collaboration at RHIC scanned the beam energies in order to search for QCD critical endpoint [5]. For the same reason, the NA61/SHINE experiment at CERN-SPS continues to search the first-order phase transition using light and medium size beams [6]. Neither NA61 at SPS nor STAR at RHIC will be able to run at beam energies of $10 \mathrm{AGeV}$ and less. At the Joint Institute for Nuclear Research in Dubna, a fixed target experiment is under preparation at the Nuclotron in order to investigate the heavy-ion collisions at gold-beam energies up to $4 \mathrm{AGeV}$. Moreover, the collider facility NICA with multi-purpose detector(MPD) is planed at JINR [7]. However, due to luminosity or detector limitations these experiments are constrained to the investigation of abundantly produced particles.

In contrast, the Compressed Baryonic Matter (CBM) experiment [1] is designed to run at extremely high interaction rates (up to $10 \mathrm{MHz}$ ) [8].

^e-mail: pkurilkin@jinr.ru 


\section{CBM detector setup}

The CBM experiment is a fixed target heavy-ion experiment. It will be set up in an underground cave to which the beam from the SIS100/300 synchrotrons will be extracted. The detector system of CBM is depicted schematically in figure 1 . The geometrical acceptance of the CBM detector systems is full $2 \pi$ for azimuth $(\phi)$ and from $2.5^{\circ}$ to $25^{\circ}$ for polar $(\Theta)$ angles. The target, Silicon Tracking System (STS) and Micro Vertex detector (MVD) are placed in the large gap of the superconducting dipole magnet [9] for charged particle tracking and the detection of short-lived decays. Downstream the beam follow the Ring Imaging Cherenkov Detector (RICH) and the Transition Radiation Detector (TRD) for electron identification and pion separation. The Time-of-Flight system identifies hadrons. The Projectile Spectator Detector (PSD) serves event plane characterization.

The CBM detector system will provide both electron and muon detection systems. Muon and electron pairs created during particle decays will be measured during separate runs in order to control the systematic approach of the measurements. In electron configuration following detectors will be used: MVD, STS, RICH, TRD, Resistive Plate Chambers for time-of-flight measurements, Electromagnetic Calorimeter (ECAL) and Projectile Spectator Detector (PSD). In muon configuration the RICH detector will be replaced by the Muon Detection System (MuCh) and ECAL will be removed.

The non-interacting beam particles, $99 \%$ of all with a typical target of $1 \%$ nuclear interaction length, are guided in a vacuum beam pipe through the experiment and are absorbed in the beam dump [8]. The HADES detector [10] will be placed in front of the CBM detector as shown in figure 1 to conduct a complementary physics program in separate data taking periods. The CBM setup may be operated in a minimal mode at FAIR startup with only key subsystems and the necessary part of the data acquisition and online computing installed, which are later on completed. Similarly, different optimizations of the experiment to perform measurements at SIS 100 and SIS 300 are possible.

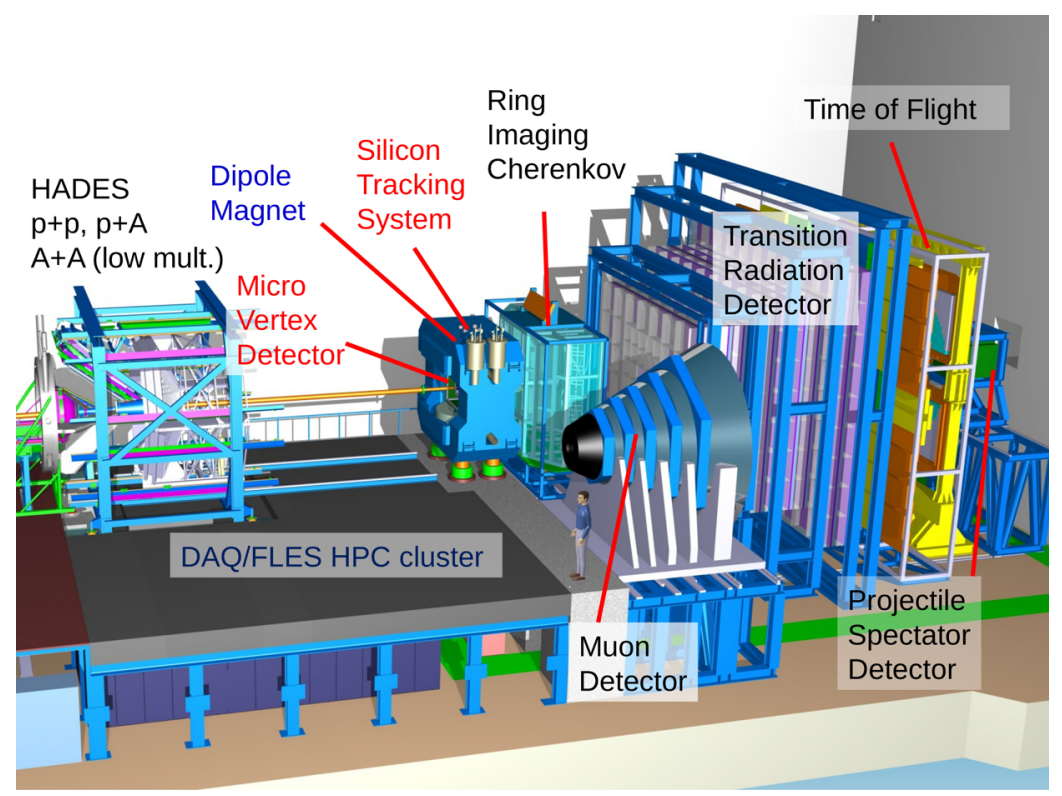

Figure 1: Schematic view of the CBM cave with the detector setup of CBM (right) and HADES (left). 


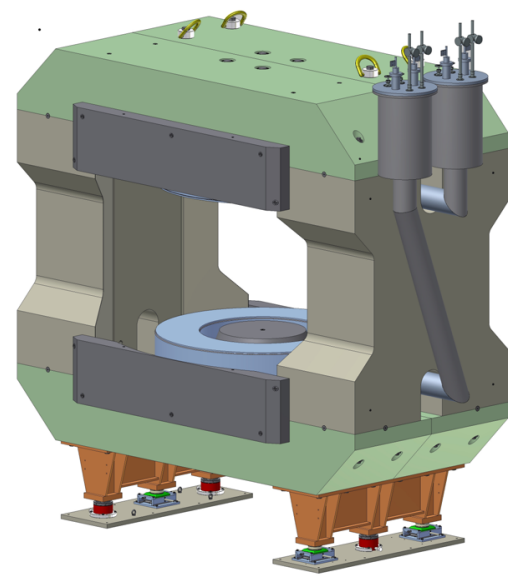

Figure 2: 3D view of CBM dipole magnet with the support.
Table 1: Parameters of the CBM dipole magnet.

\begin{tabular}{|l|l|}
\hline type & H-type, SC magnet \\
\hline Number of turns per coil & 1749 \\
\hline Windings of coil & $\begin{array}{l}\text { Impregnated } \\
\text { close coiling }\end{array}$ \\
\hline Maximum current & $686 \mathrm{~A}$ \\
\hline Magnetomotive force & $1.2 \mathrm{MA}$ turns/coil \\
\hline Current density, & $58 \mathrm{~A} / \mathrm{mm}^{2}$ \\
\hline Maximum field at coil & $3.25 \mathrm{~T}$ \\
\hline Central field & $1.08 \mathrm{~T}$ \\
\hline Field integral & $1 \mathrm{Tm}$ \\
\hline Inductance & $33-19 \mathrm{H}$ \\
\hline Stored energy & $5.15 \mathrm{MJ}$ \\
\hline Coil cross section $($ at $4 \mathrm{~K})$ & $158.8 \mathrm{x} 131.1 \mathrm{~mm}$ \\
\hline Yoke (widh/depth/height) & $4.4 / 2.0 / 3.7 \mathrm{~m}$ \\
\hline Pole type & Tapered \\
\hline Pole sizes (Rout/Rin/H) & $1200 / 800 / 500 \mathrm{~mm}$ \\
\hline
\end{tabular}

\section{CBM dipole magnet}

The H-type superconducting magnet of the CBM experiment is designed to provide a vertical magnetic field with bending power of $1 \mathrm{Tm}$ over a length of $1 \mathrm{~m}$ from the target. The maximum energy storage is evaluated as 5.15 MJ when the operating current of the CBM magnet is rated at 686 A. Calculations have been performed to design the coil case, the coil vessel, the support links and the quench protection scheme for the CBM superconducting dipole magnet [11]- [17]. The code TOSCA [18] was used for calculating electromagnetic forces exerted on the coil, while the structural analysis was made using the code ANSYS [19]. The 3D quench calculations for the CBM magnet coil have been carried out by two numerical programs based on the finite-difference method. One of them is used currently at GSI and its detailed description can be found in [20]. The other (SQUID) based on CIEMAT numerical code [21] was adapted to perform the quench simulation for the CBM magnet.

A 3D model of the magnet with the support is presented in figure 2. The parameters of the magnet are listed in table 1 . The magnet yoke consists of two conical poles and two horizontal and two vertical parts to close a flux return. Two cylindrical superconducting coils will be installed in their respective cryostats [13] and will be cooled separately by helium bath based cooling system like the SAMURAI magnet at RIKEN [22]. Each of the sections includes an inner helium vessel, thermal shield and vacuum vessel [14]. The vacuum vessel (see figure 3) has flanges for connecting to the external cryogenic line, the turbo molecular pump, two 1000 A current leads, the multipin feed through with plug connectors, the safety valve of the vessel and the warm multipurpose helium line (quench gas collection). Also, the vacuum vessel and the heat shield have a large diameter access hole to connect pipes of the external cryogenic line with helium pipes of coils. All these connections are made by means flexible metal hoses. Internal helium vessel contains 10 liters of liquid helium, and has four flanges. 


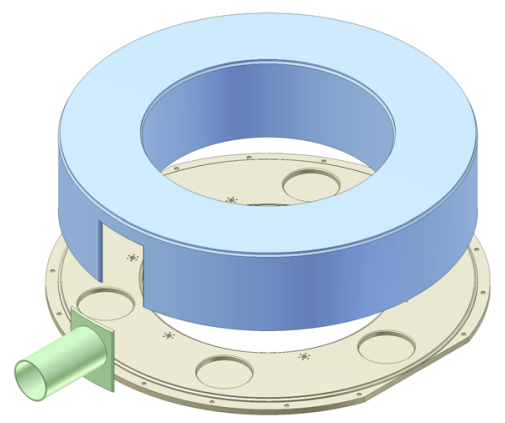

Figure 3: 3D view of the vacuum vessel.

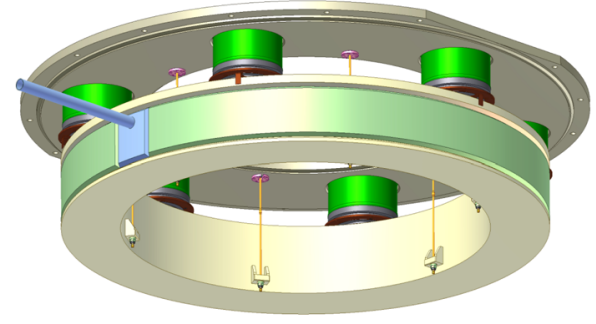

Figure 4: 3D view of the coil case support.

\subsection{Coil case and winding}

The design of coil for the CBM magnet is based on the design of the Super-FRS dipole [23]. The basic structure of the coil consists of the layer type helical winding on a stainless steel bobbin. Each coil has 1749 turns and 53 layers. The superconducting wire is a $N b / T i$ wire and its specification is determined from the quench analysis. The conductor insulation consists of $0.1 \mathrm{~mm}$ polyimide type and $0.2 \mathrm{~mm}$ glass fiber (G10) material. Each layer is additionally insulated from the others by three layers of $0.1 \mathrm{~mm}$ fiber glass fabric with epoxy resin. The epoxy resin is carefully applied during the winding to the gap between coil turns to restrict movement of the conductor.

The coil case is designed considering two main functions: one is to protect the windings against magnetic forces during operation, and the other is to use the case as a container for liquid helium (LHe) to cool the winding. The coil case is supported by six main cylindrical support strats and six tie rods. To reduce the heat flux to the helium system, the outer surface of the casing will be wrapped with 20 layers of a multi-layer insulation. The suspension during the working cycle has two types of loading. When the magnet is switched off only the weight of the cold mass is applied to the suspension. In this case the vertical force is about $2000 \mathrm{~kg}$. When the magnet is switched on, the vertical component of the Lorenz forces should be added to the weight of the cold mass. The maximum vertical force in this case is 254 tons. Figure 4 shows the suspension of the coil case on the flange of the vacuum vessel.

\subsection{Quench protection}

The 3D quench calculations [15]- [17] have been performed to optimize the behaviour of the winding of the magnet during the quench. The magnet coil is represented as a straight slab with a length equal to the average turn length. A regular mesh is applied to the slab so that the crosssection of the one mesh element equal to the cross-section of the insulated conductor. Longitudinally, the coil is divided into a number of slices which define a mesh element size. The material properties are homogeneous within each mesh element. Two possible quench protection systems for the CBM magnet were considered [17]. The final one is based on the extraction of the energy stored in the magnet via a dump resistor $(R d)$. The details can be found in [9]. The dump resistor is connected in parallel to the magnet and it is always on. In case of the quench the magnet will be disconnected from the power supply and the magnet current will be dumped via the dump resistor. The dump resistor with resistance of $1.9-2.1 \Omega$ will be used. Figure 5 presents the quench calculation results using the $2.1 \Omega$ dump resistor for the energy extraction. Simulation shows that the maximal "hot-spot" temperature in the coil in this case is about $70 \mathrm{~K}$. The maximum voltage across the magnet (1441 V) 


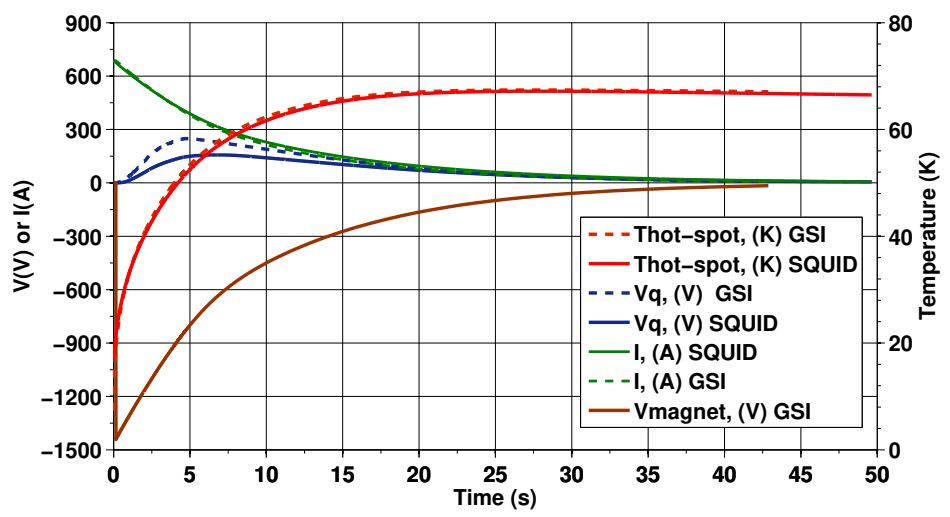

Figure 5: 3D quench calculations for the CBM magnet: magnet current, magnet voltage and the maximum ("hot-spot") temperature. A dump resistor of $R d=2.1 \Omega$ is used for quench protection.

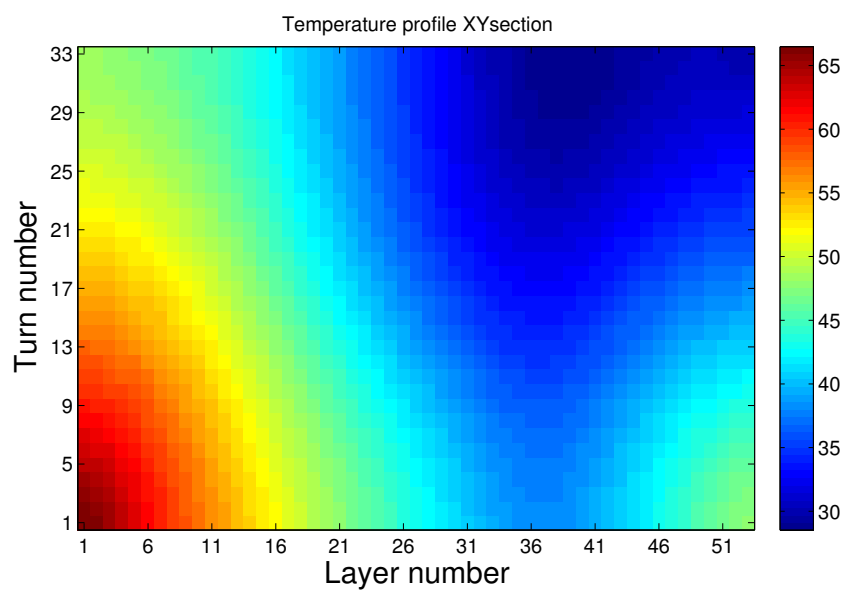

Figure 6: 3D quench calculations for the CBM magnet: the temperature distribution in the cross section of CBM magnet coil. The $85 \%$ of magnet storage energy is evacuated via $2.1 \Omega$ dump resistor.

occurs when the dump resistor turns on. The temperature profile distribution in the coil cross section when the "hot-spot" temperature has a maximum value is shown in figure 6.

\section{Conclusion}

Compressed Baryonic Matter experiment is devoted to the third generation of the heavy-ion collision experiments. Its research program aims at the exploration of the structure of high density matter including. For these purposes the advanced experimental setup will be build for high counting rate conditions expected at FAIR.

The designed superconducting dipole magnet will be a unique component of CBM detector setup 
due to a large pole gap and wide horizontal opening. Technical design report for the Superconducting Dipole Magnet has already been approved by FAIR.

The design of the CBM magnet, its support, the cryogenic input lines, current leads and feed boxes has been performed to satisfy the working conditions. The quench protection system will be based on the energy evacuation from the $2.1 \Omega$ dump resistor.

\section{Acknowledgement}

The work has been supported in part by the BMBF-JINR grant.

\section{References}

[1] Compressed Baryonic Matter (CBM) http://www.fair-center.eu/public/experimentprogram/nuclear-matter-physics/cbm.html

[2] C. Hoehne et al., J. Phys. 420, 012016 (2003)

[3] C. Pinkerburg et al., Phys. Rev. Lett. 83, 1295 (1999)

[4] C. Alt et al., Phys. Rev. C 77, 024903 (2008)

[5] A. Schmah, arXiv:1202.2389v1 (2012)

[6] A. Laszlo et al. (The NA61 Coll.), Pos(CPOD07)054 (2007)

[7] http://nica.jinr.ru

[8] E. Heuser, EPJ Web of Conferences 95, 01006 (2015)

[9] P. Akishin et al. (The CBM collaboration), Technical Design Report for the CBM Superconducting Dipole Magnet (2014)

[10] G. Agakishiev et al. (HADES collaboration), Eur. Phys. J. A 41, 243 (2009)

[11] A. Bychkov et al., CBM Progress Report 2014 (Darmstadt, 2015) 12

[12] Yu. Gusakov et al., CBM Progress Report 2015 (Darmstadt, 2016) 4

[13] Yu. Gusakov et al., CBM Progress Report 2015 (Darmstadt, 2016) 5

[14] P. Akishin et al., CBM Progress Report 2013 (Darmstadt, 2014) 5

[15] P. Kurilkin et al., CBM Progress Report 2015 (Darmstadt, 2016) 7

[16] P. Kurilkin et al., CBM Progress Report 2013 (Darmstadt, 2014) 8

[17] P. Kurilkin et al., J. Phys.: Conf. Ser. 742, 012017 (2016)

[18] Vector Fields Ltd., Tosca. 3d electromagnetics code (Kidlington, 1984)

[19] http://www.ansys.com/

[20] P. Szwangruber et al., IEEE Trans. Appl. Supercond. 23, 4701704 (2013)

[21] F. Toral, Design and Calculation Procedure for Particle Accelerator Superconducting Magnets: Application to an LHC Superconducting Quadrupole, Ph. D. Thesis. (Madrid, 2001)

[22] H. Sato et al., IEEE Trans. Appl. Supercond. 23, 4500308 (2013)

[23] L. Z. Ma et al., IEEE Trans. Appl. Supercond. 22, 9502104 (2012) 